\title{
A mathematical model to predict the performance of advanced therapies in wound healing
}

\author{
J. Ko ${ }^{1}$, S. Dickman ${ }^{2}$ \& V. W. Li ${ }^{3,4}$ \\ ${ }^{1}$ Department of Mathematics, Brown University, USA \\ ${ }^{2}$ Brown University, Providence, USA \\ ${ }^{3}$ Institute of Advanced Studies, The Angiogenesis Foundation, USA \\ ${ }^{4}$ Angiogenesis and Wound Healing Center, Department of Dermatology, \\ Brigham and Women's Hospital, Harvard Medical School, USA
}

\begin{abstract}
Wound healing is a complex, dynamic process. The ability to simulate this process using mathematical models that incorporate quantitative data on growth factors, tissue repair cells and matrix components would be a powerful tool to predict, analyze, and optimize new therapies. We present such a mathematical framework based on a system of ordinary differential equations and wound healing parameter values from the established literature. In contrast to conventional therapy, advanced modalities can augment certain components of the healing process in a measurable fashion. The performance of specific wound therapies can be simulated and compared to other therapies. We have enhanced the model by incorporating parameters of clinical practice used in the real world setting. This approach has application to predictive performance analysis and optimization of new advanced modalities and determination of best clinical practice.

Keywords: wound healing, differential equations, bifurcation.
\end{abstract}

\section{Introduction}

Wound healing is a complex process involving the interaction of many cell types and signaling molecules. In the U.S. alone, 6 million patients have chronic wounds. In many conditions, such as diabetes, the wound healing process is complicated by biochemical imbalances that often lead to wounds that do not heal. Each year, 
$5 \%$ of diabetics develop a leg ulcer, of which $53 \%$ do not heal even after 20 weeks. Several mathematical models have been proposed to predict healing behavior for normal vs. diabetic wounds. These models have been proposed to be able to estimate the effectiveness of various clinical treatments such as skin grafts and growth factor therapy. Little work has been done, however, to compare these models with clinical evidence and to ensure that the models and the parameter values accurately represent the current best clinical practices. In addition, recent clinical and laboratory research findings call for revision to previous models.

\section{Model description}

We describe here a general framework for a wound healing system based on the models of Waugh and Sherratt. Each of the numerous variables in the wound healing process - cell types, matrix components, growth factors, and other signaling molecules - are modeled as concentrations over time. Adapting the classic predator-prey population model, the rate of change of a cell population is modeled by the rate of cell migration, cell mitosis and cell death. Similarly, the rate of change of a growth factor, matrix component or chemical mediator population is modeled by the rate of production minus the rate of decay.

The basic mathematical model in [1] is given by the following system of equations:

$$
\left\{\begin{array}{l}
\dot{\phi_{I}}=\alpha K(T)+k_{1} k_{2} \phi_{I}\left(1-k_{3}\left(\phi_{I}+\phi_{R}\right)\right)-d_{1} \phi_{I} \\
\dot{\phi_{R}}=(1-\alpha) K(T)+k_{1} k_{2} \phi_{R}\left(1-k_{3}\left(\phi_{I}+\phi_{R}\right)\right)-d_{1} \phi_{R} \\
\dot{T}=k_{4} \phi_{I}-d_{2} T
\end{array}\right.
$$

where the variables $\phi_{I}, \phi_{R}$ and $T$ are the densities of inflammatory macrophages, repair macrophages and the growth factor TGF- $\beta$, respectively; $k_{i}, d_{1}$ are growth and decay rates of the variables; $K(T)$ is the effect of monocyte migration due to TGF- $\beta$ as seen in controlled-interaction studies; $\alpha \in[0,1]$ is the fraction of monocytes becoming inflammatory macrophages and will also be seen to be a bifurcation parameter for this system.

This system attempts to capture an essential part of the wound healing process, as illustrated in Figure 1. Significantly, the system uses a surrogate endpoint of healing- the "zero equilibrium point"- at which the inflammatory macrophage population has stabilized to its pre-wound level. This zero equilibrium point generally corresponds to the cessation of wound inflammation and proliferative activity that accompanies closure of the wound cavity and surface. The contributions of epidermal keratinocytes, platelets, and blood vessel endothelial cells - ignored in previous models - can be captured their impact on TGF- $\beta$, fibroblasts and PDGF.

A bifurcation analysis of this model shows that there exists an $\alpha^{*}$ such that for $\alpha \in\left[0, \alpha^{*}\right)$, there is one stable equilibrium and for $\alpha \in\left[\alpha^{*}, 1\right]$, there are three equilibria, with a sequence of stable-unstable-stable. This analysis underlies the initial choice of $\alpha$ in the model: an initial choice of $\alpha \in\left[0, \alpha^{*}\right)$ corresponds to 


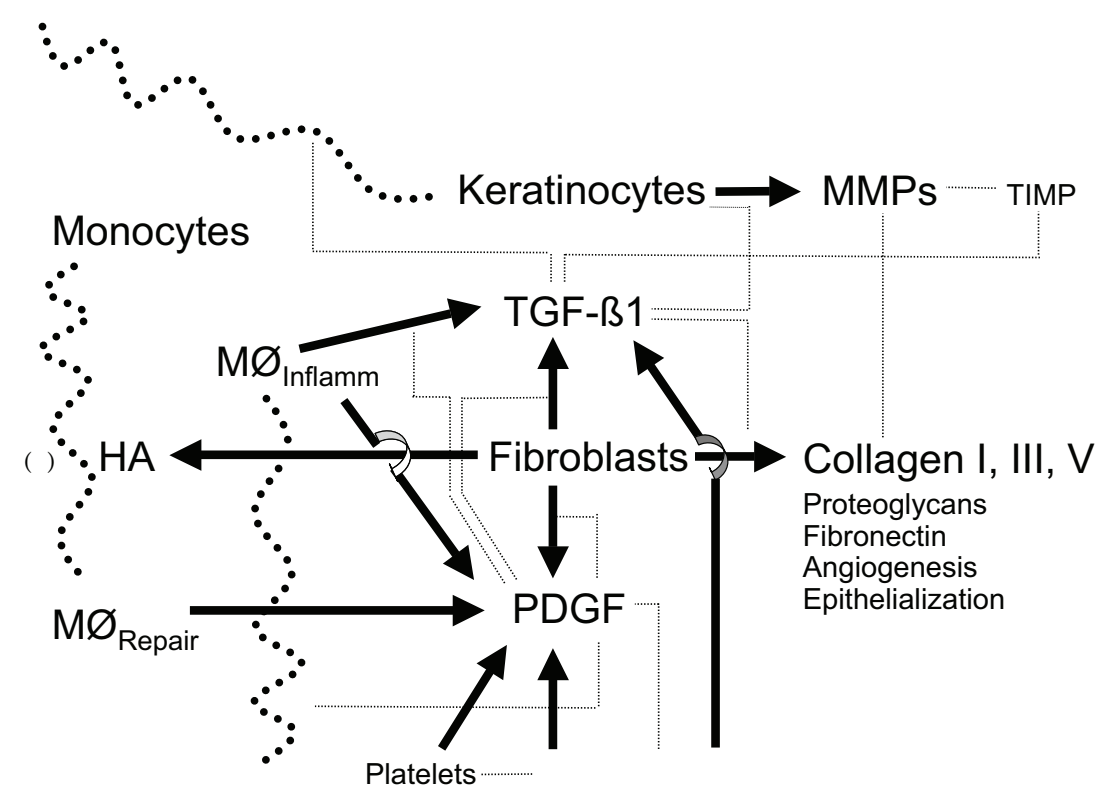

Figure 1: Modeled interactions in the wound healing process. Adapted from [2].

wound healing in normal skin and $\alpha \in\left[\alpha^{*}, 1\right]$ corresponds to healing in diabetic skin.

A subsequent 7-variable revision in [3] builds upon this basic model, with the additional variables of the densities of fibroblasts $(F)$, hyaluronan $(H A)$, collagen $(C)$, and PDGF $(P)$. An equilibrium analysis shows a similar bifurcation structure as in the simplified model (1). Fixing a value of $\alpha_{\text {normal }}=0.5$ and $\alpha_{\text {diabetic }}=0.8$, and substituting all parameter values, we can solve for the equilibrium points $\left(\phi_{I}, \phi_{R}, T, P, F, C, H\right)$. In both cases, we found more than 100 equilibrium points. Most equilibrium points were disregarded because they contained complex coordinates and were thus nonphysical. Moreover, equilibrium points with negative coordinates could also be disregarded, as these too represent a nonphysical representation. Restricting to real-valued equilibrium points, a linear analysis readily shows that the situation is similar to that of (1): for $\alpha=\alpha_{\text {normal }}$, the one real-valued equilibrium is stable, and for $\alpha=\alpha_{\text {diabetic }}$, three real-valued equilibria exist, with the sequence being stable-unstable-stable.

A persistent feature of this revised model is the relative magnitude of the quantities $\phi_{I}, \phi_{R}, T$ at each of the equilibrium points in the diabetic case relative to magnitude at the normal state. The first diabetic equilibrium point has coordinate values similar to those of the stable equilibrium point in the normal case. This first equilibrium point shows that diabetic wounds are capable of reaching a healing state. The second equilibrium point corresponds to the chronic inflammation state. The last equilibrium point, which has elevated levels of macrophages $\phi_{I}, \phi_{R}$ 
(where $\phi_{I} \gg \phi_{R}$ ), $T$, and $P$ relative to the levels at equilibrium in the normal case, corresponds to a chronic non-healing wound.

In this paper, we describe a model which reflects ongoing clinical research findings. Our model is a system which incorporates the effect of suppressed levels of PDGF in diabetic wounds and the presence of epidermal keratinocytes in one of the skin graft therapies (Apligraf) by modeling its effect on TGF- $\beta$. Importantly, we show how data from controlled experiments can be incorporated into this model in lieu of modeling the evolution of a variable for which only limited information is known. Our model is given by the following system of equations:

$$
\left\{\begin{array}{l}
\dot{\phi}_{I}=\alpha K(T)+k_{1} k_{2} \phi_{I}\left(1-k_{3}\left(\phi_{I}+\phi_{R}\right)-k_{5} F-k_{6} C\right)-d_{1} \phi_{I} \\
\dot{\phi_{R}}=(1-\alpha) K(T)+k_{1} k_{2} \phi_{R}\left(1-k_{3}\left(\phi_{I}+\phi_{R}\right)-k_{5} F-k_{6} C\right)-d_{1} \phi_{R} \\
\dot{T}=k_{4} \phi_{I}+k_{7} K_{C}(t) F-d_{2} T \\
\dot{P}=k_{8}(\alpha)\left(\phi_{I}+\phi_{R}\right)+k_{9} F-d_{3} P \\
\dot{F}=M(P)+k_{10} F\left(1-k_{3}\left(\phi_{I}+\phi_{R}\right)-k_{5} F-k_{6} C\right)-d_{4} F \\
\dot{C}=k_{11} F+F f(T) g(C)-d_{5} F C \\
\dot{H}=k_{12} F-d_{6} H
\end{array}\right.
$$

where $k_{i}, d_{i}$ refer to growth and decay rates, $M(P), f(T), g(C)$ are measured migration effects as seen in controlled-interaction studies, and $K_{C}$, which is nonzero only for the treatment Apligraf, is the measured effect of keratinocytes on TGF- $\beta$ in the presence of fibroblasts. Exact parameter values used in the simulations are provided in the Appendix.

\section{Testing the model against clinical data}

As an initial test for our model, we compared data from wound healing clinical trials with predictions made by our wound healing model.

\subsection{Topical growth factor therapy for diabetic ulcers}

One clinical trial was designed to examine the effectiveness of rhPDGF-BB $0.01 \%$ gel (becaplermin, Systagenix, London), a topical growth factor therapy which dramatically increases local PDGF levels at the wound site, in treating diabetic ulcers. The ulcers had been unhealed for at least 60 days before treatment began. One group of patients was treated with the medication daily for 20 weeks, while a control group was given placebo control. Applying these conditions, our model predicts that wounds in the control group remains at an unhealed steady state, while the wounds receiving the topical growth factor reaches the stable equilibrium in 23 weeks (Figure 2). This is in good agreement to the results of the clinical trial, in which patients receiving medication healed significantly $(P=0.01)$ faster than the control group. Roughly $50 \%$ of patients receiving medication were healed within 

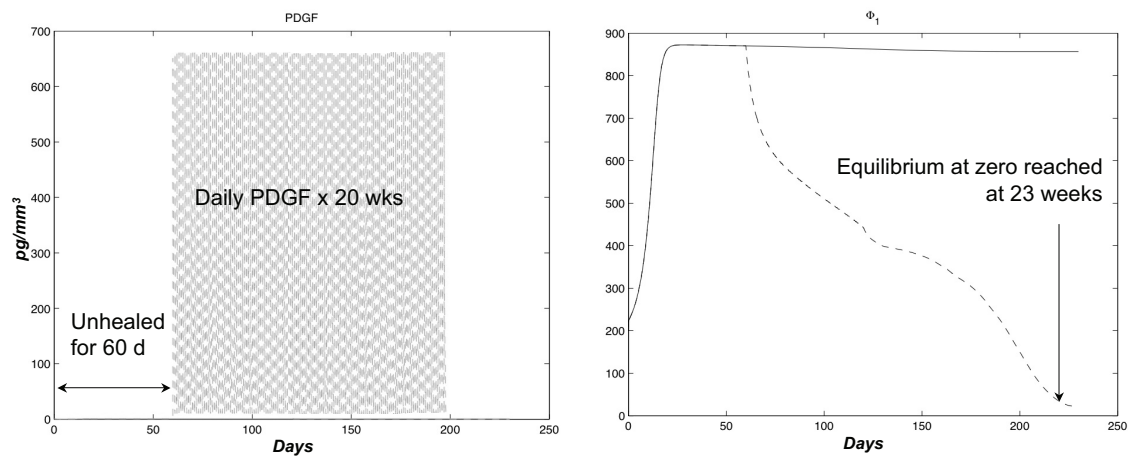

Figure 2: Topical PDGF therapy for chronic diabetic ulcers. Left: dosing; right: time to surrogate endpoint for healing.
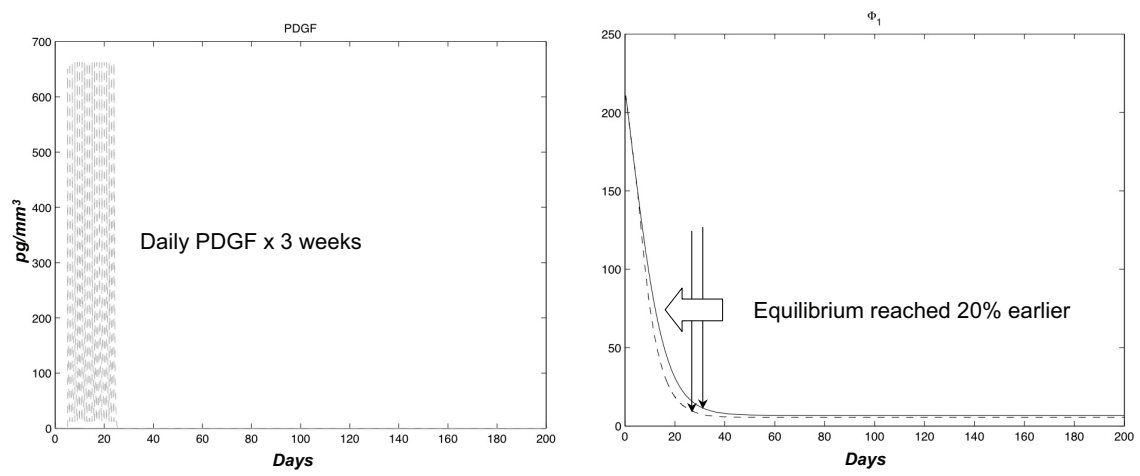

Figure 3: Topical PDGF therapy for acute wounds.

20 weeks, compared to $25 \%$ of those in the control group, demonstrating a $43 \%$ increase in the incidence of healing $(P=0.007)$ [4].

\subsection{Topical growth factor therapy for acute wounds}

Another clinical trial was undertaken to examine the effectiveness of PDGF growth factor therapy (becaplermin gel) on acute, non-diabetic wounds, compared to the topical antibiotic bacitracin. Participants in the experimental group received daily applications of PDGF for three weeks immediately following the wound event. Figure 3 shows the prediction of our model: the group receiving growth factor therapy achieves equilibrium $20 \%$ earlier than the control group. Clinical data, by comparison, showed that growth factor therapy helped achieve complete wound closure $32 \%$ faster than bacitracin [5]. 


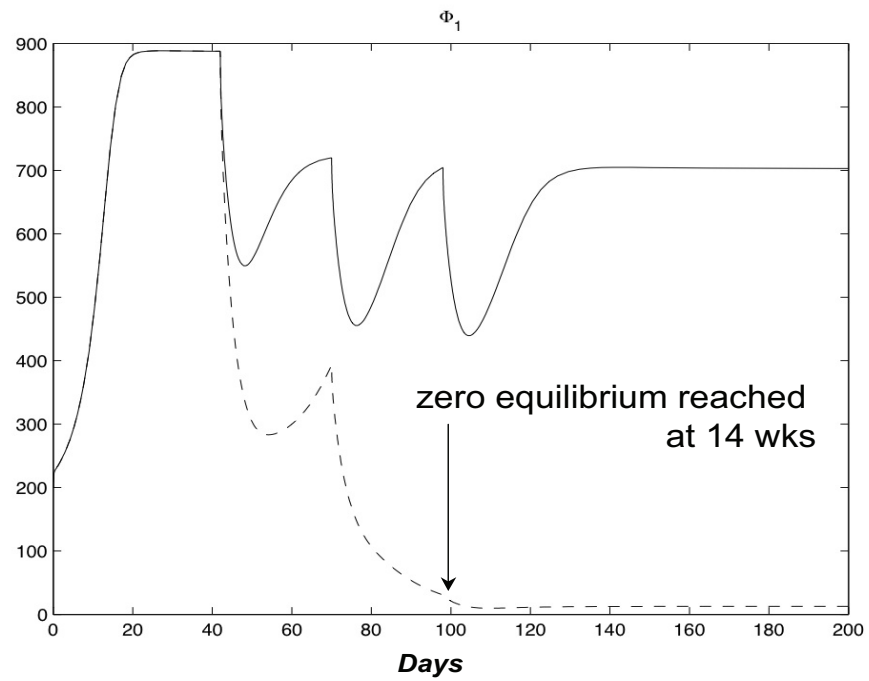

Figure 4: Bioengineered cryopreserved dermal fibroblast therapy for chronic diabetic ulcers and time to surrogate endpoint for healing.

\subsection{Dermagraft therapy for diabetic ulcers}

A clinical trial was designed to test the effectiveness of Dermagraft (Advanced BioHealing, Westport, CT), a bioengineered cryopreserved dermal fibroblast skin construct used to treat chronic diabetic wounds. In this trial, patients with diabetic foot ulcers which had remained unhealed for at least six weeks were randomized into a control group and a treatment group receiving Dermagraft. Figure 4 shows the prediction of our model: patients receiving Dermagraft treatment reach the surrogate healing endpoint at week 14 of treatment. In comparison, the clinical trial data showed that after 12 weeks of treatment, $30 \%$ of patients receiving Dermagraft had completely healed, compared to $18.3 \%$ of patients in the control group [6].

\subsection{Apligraf therapy for diabetic ulcers}

Apligraf (Graftskin, Organogenesis, Canton, MA) is a bioengineered, cultured, bilayered living skin construct consisting of both epidermal keratinocytes and dermal fibroblasts. A clinical trial was conducted to examine the effectiveness of Apligraft treatment on diabetic foot ulcers. In our model, Apligraf was applied monthly over three months, following current best clinical practice, to ulcers which had been unhealed for at least six weeks. In this case, it is noteworthy to compare our results with those using the Waugh-Sherratt model in [3], which applied Apligraf weekly, not monthly and did not take into consideration the contribu- 

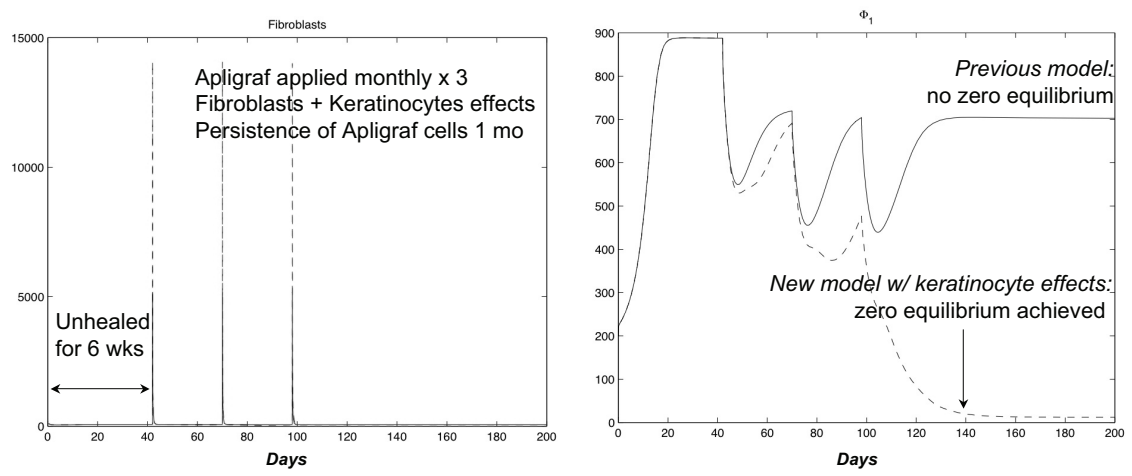

Figure 5: Bioengineered cultured bilayered skin therapy for chronic diabetic ulcers. Left: dosing of Apligraf; right: comparison of results predicted by previous model [3] and our model.

tion of epidermal keratinocytes in Apligraf. These differences may account for the previous model's inability to predict wound closure using Apligraf, instead achieving an unhealed steady state. Our model, taking into account the effect of keratinocytes on TGF- $\beta$, predicts that the zero equilibrium point is reached after 140 days (Figure 5). These results are consistent with actual clinical data. The pivotal trial showed that with three monthly applications, $56 \%$ of patients receiving Apligraft treatment had achieved complete wound closure at 12 weeks ( 84 days), compared to $39 \%$ in the control group, a $P=0.0026$ difference [7]. A separate study found that Apligraft treatment led to $70 \%$ of patients achieving complete wound healing with an average of two applications [8].

\section{Discussion and future work}

Our wound healing model uses a population-based system of equations to predict the level of certain cell types and chemicals as functions of time. By comparing predictions from the model with clinical data, we have shown that the model captures certain key features of the wound healing process. By looking at current clinical research findings, we were able to improve how the model predicts the behavior of specific treatments on healing. This type of calibration is critical and should be an ongoing process in light of new research findings. In addition, a thorough review of all equation coefficients and parameters was undertaken to increase the accuracy of the model's predictions. The system does not model the spatial distribution of tissue cells within the healing wound. Thus, the effect of cells such as epidermal keratinocytes are manifest in its influence on TGF- $\beta$ levels.

Multiple factors may impair the wound healing process. For instance, proteolysis can be stimulated by bacterial infection. In turn, excessive enzymatic proteoly- 
sis will disrupt the healing process. Other factors which slow the healing process include cellular senescence, sustained inflammation, moisture imbalance, physical pressure, compromised patient status, impaired perfusion, insufficient angiogenesis, and deficient growth factors [9]. The deficiencies of growth factors like PDFG and TGF- $\beta$ can also impair wound healing.

All mathematical models which attempt to approximate a complex biological process like wound healing have limitations. Not all the variables included in this model have known accurate values. In addition, the heterogeneity between individual patients is a difficult factor to incorporate into a mathematical model. Future planned work are: (i) continually improve the accuracy of the parameter values as new data emerges from scientific and clinical studies; (ii) to introduce more variables into the model that can better represent the complexity of wound healing (eg. MMP, other growth factors); (iii) address directly the endpoint of healing via the spatio-temporal dynamics.

In summary, this model can provide important insights into the fundamental drivers that affect wound healing. This model can also be used to understand how perturbations in certain parameters can optimize healing. A practical use for this model might eventually be to guide the rational design of drug and device prior to significant investment in commercial research and development.

\section{Appendix A}

\section{A.1 Variables}

The variables in this model are the same as those used in [3], and are described below:

$\phi_{I}$ : Inflammatory macrophages $\left(\right.$ cells $\left./ \mathrm{mm}^{3}\right)$

$\phi_{R}:$ Repair macrophages (cells $/ \mathrm{mm}^{3}$ )

$\alpha$ : Proportion of migrating monocytes differentiating into $\phi_{I}$ (cells $/ \mathrm{mm}^{3}$ )

$k_{1}$ :\% macrophages undergoing mitosis

$k_{2}$ : macrophages growth rate

$k_{3}$ : inverse max macrophage density $\left(/\right.$ cells $\left./ \mathrm{mm}^{3}\right)$

$k_{4}$ : macrophage TGF- $\beta$ production rate

$K(T)$ : monocyte migration due to TGF- $\beta$

$P$ : PDGF $\left(\mathrm{pg} / \mathrm{mm}^{3}\right)$

$d_{3}$ : PDGF decay rate(/day)

$T:$ TGF- $\beta\left(\mathrm{pg} / \mathrm{mm}^{3}\right)$

$k_{7}$ : TGF- $\beta$ production rate by fibroblasts (pg/cells/day)

$K_{C}(t)$ : scaled effect of keratinocytes on TGF- $\beta$ in the presence of fibroblasts

$k_{4}$ : TGF- $\beta$ production rate by macrophages (pg/cells/day)

$d_{2}$ : TGF- $\beta$ decay rate (/day)

$F$ : fibroblasts (cells $/ \mathrm{mm}^{3}$ )

$k_{10}$ : fibroblast growth rate (/day)

$d_{4}$ : fibroblast death rate (/day)

$k_{5}$ : inverse max fibroblast density $\left(/\right.$ cells $\left./ \mathrm{mm}^{3}\right)$ 


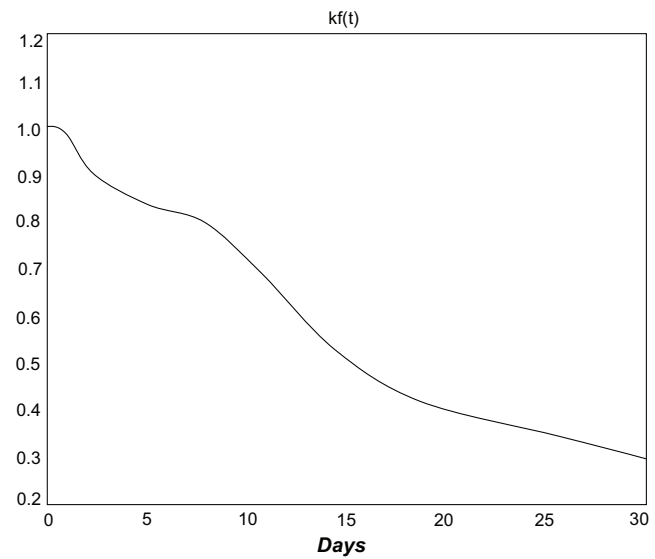

Figure 6: $k f(t)$, the measured effect of keratinocytes on TGF- $\beta$ in the presence of fibroblasts.

$k_{9}$ : fibroblast PDGF production rate (pg/cells/day)

$k_{8}$ : macrophage PDGF production rate ( $\left.\mathrm{pg} / \mathrm{cells} / \mathrm{day}\right)$

$M(P)$ : fibroblast migration due to PDGF

$C$ : collagen $\left(\mu \mathrm{g} / \mathrm{mm}^{3}\right)$

$k_{11}$ : fibroblast collagen rate ( $\left.\mu \mathrm{g} / \mathrm{cells} / \mathrm{day}\right)$

$f(T)$ : fibroblast synthesis of collagen due to TGF- $\beta$

$g(C)$ : collagen synthesis due to collagen density

$k_{6}$ : inverse max collagen density $\left(\mu \mathrm{g} / \mathrm{mm}^{3}\right)$

$d_{5}$ : collagen remodeling rate (/day)

$H$ : hyaluronan

$k_{12}$ : hyaluronan synthesis by fibroblasts ( $\left.\mu \mathrm{g} / \mathrm{cells} / \mathrm{day}\right)$

$d_{6}$ : hyaluronan decay rate (/day)

\section{A.2 Determination and refinement in parameter values}

We describe here any changes to parameters that were also used in [3] as well as the determination of new parameter values in our model.

- Start treatment time points are defined at a point where the wound has become chronic (e.g. one month $=28$ days), which is the inclusion criteria for most clinical trials in wound healing.

- $k_{8}=k_{8}(\alpha)$ : Recent research in [10] shows that diabetic limb tissues are deficient in the growth factor PDGF by up to $40 \%$.

- The number of fibroblasts and collagen in Apligraf and Dermagraft were determined based on the fibroblast density and thickness of the product, according to personal communication with Katie Faria, Organognesis and Gary Gentzkow (Advanced Tissue Sciences) 
Table 1: Parameter values used in simulations.

\begin{tabular}{|c|c|c|c|c|c|c|}
\hline Variable & Initial & Normal & Diabetic & Apligraf & Dermagraft & Regranex \\
\hline$\phi_{I}$ & 200 & & & & & \\
\hline$\phi_{R}$ & 200 & & & & & \\
\hline$\alpha$ (initial) & & 0.5 & 0.8 & & & \\
\hline$k_{1}$ & & 0.05 & 0.05 & & & \\
\hline$k_{2}$ & & 0.693 & 0.693 & & & \\
\hline$k_{3}$ & & 0.002 & 0.002 & & & \\
\hline$k_{4}$ & & 0.07 & 0.07 & & & \\
\hline$d_{1}$ & & 0.2 & 0.2 & & & \\
\hline$P$ & 2 & & & 1 & 1 & 650 \\
\hline$d_{3}$ & & 4.0 & 4.2 & & & \\
\hline$T$ & 6 & & & 4 & 0.4 & \\
\hline$k_{7}$ & & 0.004 & 0.004 & & & \\
\hline$k_{4}$ & & 0.07 & 0.07 & & & \\
\hline$d_{2}$ & & 9.1 & 9.1 & & & \\
\hline$\overline{F F}$ & 6 & & & 25100 & 14000 & \\
\hline$k_{10}$ & & 0.924 & 0.924 & & & \\
\hline$d_{4}$ & & 1.0 & 2.5 & & & \\
\hline$k_{5}$ & & 0.0025 & 0.0025 & & & \\
\hline$k_{9}$ & & 0.0015 & 0.0015 & & & \\
\hline$k_{8}$ & & 0.015 & 0.003 & & & \\
\hline $\bar{C}$ & 10 & 2.5 & 0.015 & 9.1 & 8.5 & \\
\hline$k_{11}$ & & 20 & 5 & & & \\
\hline$k_{6}$ & & 0.0004 & 0.0004 & & & \\
\hline$d_{5}$ & & $1.50 \mathrm{e}-05$ & $1.50 \mathrm{e}-05$ & & & \\
\hline$H$ & 0.01 & & & 7.45 & 80 & \\
\hline$d_{6}$ & & 0.7 & 0.7 & & & \\
\hline$k_{12}$ & & 0.008 & 0.001 & & & \\
\hline
\end{tabular}

- The effect of PDGF on fibroblast migration was determined by calculating cells per field (400x magnification) in studies [11].

- The concentration of PDGF was determined through personal communication with Liza Ovington, Johnson \& Johnson Wound Management.

- $K_{C}(t)$ : the presence of keratinocytes in one product (Apligraf) is taken into consideration through its effect on suppressed TGF- $\beta$ production by fibrob- 
lasts in studies of co-cultures [12]. To find $K_{C}(t)$, we take the curve $k f=$ $k f(t)$ derived from the curve that measures the effect of keratinocytes and fibroblasts on TGF- $\beta$ normalized by the base curve that measures the effect of fibroblasts alone over the course of 30 days. The curve for $k f$ used in our simulations is shown in Figure 6. $K_{C}(t)$ can be taken to be:

$$
K_{C}(t)=\left((k f)(t)+\frac{1}{k_{7}}(k f)^{\prime}(t)\right) e^{k_{7} * t}
$$

In our simulations, we take the variable functions $\alpha(H), K(T), M(P), f(T)$ and $g(C)$ to be the same as in [3].

\section{Acknowledgement}

Many thanks to Jonathan Sherratt for his interest, his expertise, and his generosity.

\section{References}

[1] Waugh, H.V. \& Sherratt, J.A., Macrophage dynamics in diabetic wound healing. Bull Math Biol, 68(1), pp. 197-207, 2006.

[2] Pierce, G.F., Mustoe, T.A. \& Lingelbach, J.et al.., Platelet-derived growth factor and transforming growth factor-beta enhance tissue repair activities by unique mechanisms. J Cell Biol, 109, pp. 429-440, 1989.

[3] Waugh, H.V. \& Sherratt, J.A., Modelling the effects of treating diabetic wounds with engineered skin substitutes. Wound Rep Reg, 15(1), pp. 556565, 2007.

[4] Wieman, T., Smiell, J. \& Su, Y., Efficacy and safety of a topical gel formulation of recombinant human platelet-derived growth factor-BB (becaplermin) in patients with chronic neuropathic diabetic ulcers. a phase iii randomized placebo-controlled double-blind study. Diabetes Care, 21(5), pp. 822-827, 1998.

[5] Li, V.W., Ma, J. \& Ko, J.et al.., Dynamics of acute wound healing following topical rhPDGF therapy. J Am Acad Dermatol, 52(3), p. P213, 2005.

[6] Marston, W.A., Hanft, J., Norwood, P. \& Pollak, R., The efficacy and safety of Dermagraft in improving the healing of chronic diabetic foot ulcers: results of a prospective randomized trial. Diabetes Care, 26, pp. 1701-1705, 2003.

[7] Veves, A., Falanga, V., Armstrong, D.G. \& et al., Graftskin, a human skin equivalent, is effective in the management of noninfected neuropathic diabetic foot ulcers: a prospective randomized multicenter clinical trial. Diabetes Care, 24, pp. 290-295, 2001.

[8] Novartis US08 Non-Controlled Study. Unpublished.

[9] CMS Medical Coverage Advisory Committee Meeting on Usual Care of Chronic wounds. Baltimore, MD, 2005.

[10] Tanii, M., Yonemitsu, Y. \& Fujii, T.et al.., Diabetic microangiopathy in ischemic limb is a disease of disturbance of the platelet-derived growth 
246 Modelling in Medicine and Biology VIII

factor-BB/protein kinase $\mathrm{C}$ axis but not of impaired expression of angiogenic factors. Circ Res, 98, pp. 55-62, 2006.

[11] Facchiano, A., De Marchis, F. \& Turchetti, E.et al.., The chemotactic and mitogenic effects of platelet-derived growth factor-BB on rat aorta smooth muscle cells are inhibited by basic fibroblast growth factor. J Cell Sci, 113 (Pt 16), pp. 2855-2863, 2000.

[12] Le Poole, I.C. \& Boyce, S.T., Keratinocytes suppress transforming growth factor-beta1 expression by fibroblasts in cultured skin substitutes. $\mathrm{Br} J \mathrm{Der}$ matol, 140, pp. 409-416, 1999. 